\title{
ON A SYSTEM OF NONLINEAR WAVE EQUATIONS OF KIRCHHOFF TYPE WITH A STRONG DISSIPATION
}

\section{\|\|}

\author{
SHUN-TANG WU AND LONG-YI TSAI
}

\begin{abstract}
The initial boundary value problem for systems of nonlinear wave equations of Kirchhoff type with strong dissipation in a bounded domain is considered. We prove the local existence of solutions by Banach fixed point theorem and blow-up of solutions by energy method. Some estimates for the life span of solutions are given.
\end{abstract}

\section{Introduction}

We consider the initial boundary value problem for the following nonlinear coupled wave equations of Kirchhoff type :

$$
\begin{gathered}
u_{t t}-M\left(\|\nabla u\|_{2}^{2}\right) \Delta u-\Delta u_{t}=f_{1}(u, v) \quad \text { in } \Omega \times[0, \infty), \\
v_{t t}-M\left(\|\nabla v\|_{2}^{2}\right) \Delta v-\Delta v_{t}=f_{2}(u, v) \quad \text { in } \Omega \times[0, \infty),
\end{gathered}
$$

with initial conditions,

$$
\begin{aligned}
& u(x, 0)=u_{0}(x), u_{t}(x, 0)=u_{1}(x), \quad x \in \Omega, \\
& v(x, 0)=v_{0}(x), v_{t}(x, 0)=v_{1}(x), \quad x \in \Omega,
\end{aligned}
$$

and boundary conditions,

$$
\begin{aligned}
& u(x, t)=0, x \in \partial \Omega, t>0, \\
& v(x, t)=0, x \in \partial \Omega, t>0,
\end{aligned}
$$

where $\Omega \subset R^{N}, N \geq 1$, is a bounded domain with smooth boundary $\partial \Omega$ so that Divergence theorem can be applied. Let $\Delta=\sum_{j=1}^{N} \frac{\partial^{2}}{\partial x_{j}^{2}}$ be the Laplace operator, and $M(r)$ be a nonnegative locally Lipschitz function for $r \geq 0$ like $M(r)=a+b r^{\gamma}$, with $a \geq 0$, $b>0, a+b \geq 0, \gamma \geq 0$, and $f_{i}(u, v), i=1,2$, be a nonlinear function. We denote $\|\cdot\|_{p}$ to be $L^{p}$-norm.

The existence and nonexistence of solutions for a single wave equation of Kirchhoff type :

$$
u_{t t}-M\left(\|\nabla u\|_{2}^{2}\right) \Delta u+g\left(u_{t}\right)=f(u) \text { in } \Omega \times[0, \infty),
$$

Received May 20, 2004; revised December 2, 2004.

2000 Mathematics Subject Classification. 35L70, 35L15, 65M60.

Key words and phrases. Blow-up, life span, dissipation, nonlinear wave equations. 
have been discussed by many authors and the references cited therein. The function $g$ in (1.7) is considered in three different cases. For $g\left(u_{t}\right)=\delta u_{t}, \delta>0$, the global existence and blow-up results can be found in $[4,10,16,22]$; for $g\left(u_{t}\right)=-\Delta u_{t}$, some global existence and blow-up results are given in $[8,10,14,17,18,22]$; for $g\left(u_{t}\right)=\left|u_{t}\right|^{m} u_{t}, m>0$, the main results of existence and blow-up are in $[2,3,13,15,22]$. As a model it describes the nonlinear vibrations of an elastic string. More precisely, we have

$$
\rho h \frac{\partial^{2} u}{\partial t^{2}}=\left\{p_{0}+\frac{E h}{2 L} \int_{0}^{L}\left(\frac{\partial u}{\partial x}\right)^{2} d x\right\} \frac{\partial^{2} u}{\partial x^{2}}+f,
$$

for $0<x<L, t \geq 0$; where $u$ is the lateral deflection, $x$ the space coordinate, $t$ the time, $E$ the Young modulus, $\rho$ the mass density, $h$ the cross section area, $L$ the length, $p_{0}$ the initial axial tension and $f$ the external force. Kirchhoff [11] was the first one to study the oscillations of streched strings and plates, so that (1.8) is named the wave equation of Kirchhoff type. Moreover, (1.8) is called a degenerate equation when $p_{0}=0$ and nondegenerate one when $p_{0}>0$. For the system of wave equations with no dissipative terms, when $M=1$ many authors have discussed the local, global existence and blow-up properties in $[5,6,7,12]$. When $M$ is not a constant, Park and Bae [19, 20] considered the system of wave equations with $f_{1}(u, v)=|u|^{\beta} u, f_{2}(u, v)=|v|^{\beta} v, \beta \geq 0$, and showed the global existence and asymptotic behavior of solutions under some restrictions on initial energy. Later, Benaissa and Messaoudi [3] studied blow-up properties for negative initial energy.

In this paper, we investigate the local existence, blow-up properties of solutions for some nonlinear coupled wave equations of Kirchhoff type (1.1)-(1.6) in a bounded domain $\Omega$ with more general $f_{i}(u, v), i=1,2$. The paper is organized as follows. In section 2 , we present the preliminaries and some lemmas. In section 3 , we will show the existence of a unique local weak solution $(u, v)$ of our problem (1.1)-(1.6) with $u_{0}, v_{0} \in H^{2}(\Omega) \cap H_{0}^{1}(\Omega)$ and $u_{1}, v_{1} \in L^{2}(\Omega)$ by applying the Banach contraction mapping principle. In section 4 , we first define an energy function $E(t)$ by (4.1) and show that it is a nonincreasing function. Then by using the direct method [12], we obtain Theorem 4.4, which shows blow-up properties of solutions under some restrictions even for positive initial energy, that is, we prove that there exists a finite time $T^{*}>0$ such that $\lim _{t \rightarrow T^{*-}} \int_{\Omega}\left(|\nabla u|^{2}+|\nabla v|^{2}\right) d x=\infty$. Estimates for the blow-up time $T^{*}$ are also given in Remark 4.5.

\section{Preliminaries}

Let us begin by stating the following lemmas, which will be used later.

Lemma 2.1.(Sobolev-Poincaré)[17, p.154]) Let $0<p \leq \frac{2 N}{N-2 m}(0<p<\infty$ if $N=2 m)$. Then, the inequality

$$
\|v\|_{p} \leq c_{*}\left\|(-\Delta)^{\frac{m}{2}} v\right\|_{2}
$$


holds with some constant $c_{*}$.

Lemma 2.2. Let $\delta>0$ and $B(t) \in C^{2}(0, \infty)$ be a nonnegative function satisfying

$$
B^{\prime \prime}(t)-4(\delta+1) B^{\prime}(t)+4(\delta+1) B(t) \geq 0 .
$$

If

$$
B^{\prime}(0)>r_{2} B(0)+K_{0}
$$

then

$$
B^{\prime}(t)>K_{0}
$$

for $t>0$, where $K_{0}$ is a constant, $r_{2}=2(\delta+1)-2 \sqrt{(\delta+1) \delta}$ is the smallest root of the equation

$$
r^{2}-4(\delta+1) r+4(\delta+1)=0 .
$$

Proof. see [12].

Lemma 2.3. If $J(t)$ is a nonincreasing function on $\left[t_{0}, \infty\right)$ and satisfies the differential inequality

$$
J^{\prime}(t)^{2} \geq a+b J(t)^{2+\frac{1}{\delta}},
$$

where $a>0, b \in R$, then there exists a finite time $T^{*}$ such that

$$
\lim _{t \rightarrow T^{*-}} J(t)=0
$$

and the upper bound of $T^{*}$ is estimated respectively by the following cases:

(i) If $b<0$ and $J\left(t_{0}\right)<\min \{1, \sqrt{-a / b}\}$, then

$$
T^{*} \leq t_{0}+\frac{1}{\sqrt{-b}} \ln \frac{\sqrt{\frac{a}{-b}}}{\sqrt{\frac{a}{-b}}-J\left(t_{0}\right)} .
$$

(ii) If $b=0$, then

$$
T^{*} \leq t_{0}+\frac{J\left(t_{0}\right)}{J^{\prime}\left(t_{0}\right)}
$$

(iii) If $b>0$, then

$$
T^{*} \leq \frac{J\left(t_{0}\right)}{\sqrt{a}}
$$

or

$$
T^{*} \leq t_{0}+2^{\frac{3 \delta+1}{2 \delta}} \frac{\delta c}{\sqrt{a}}\left\{1-\left[1+c J\left(t_{0}\right)\right]^{\frac{-1}{2 \delta}}\right\}
$$

where $c=\left(\frac{a}{b}\right)^{2+\frac{1}{\delta}}$.

Proof. see [12]. 


\section{Local existence}

In this section we shall discuss the local existence of solutions for (1.1)-(1.6) by method of Banach fixed point theorem under the following assumptions on $f_{i}(u, v), i=$ 1,2 . In the sequal, for the sake of simplicity we will omit the dependence on $t$, when the meaning is clear.

(A1) $f_{i}: R^{2} \rightarrow R$ is continuously differentiable such that for each $(u, v) \in H_{0}^{1}(\Omega) \times$ $H_{0}^{1}(\Omega)$, we have $u f_{1}, v f_{2} \in L^{1}(\Omega)$, and $F(u, v) \in L^{1}(\Omega)$, where

$$
F(u, v)=\int_{0}^{u} f_{1}(s, v) d s+\int_{0}^{v} f_{2}(0, s) d s .
$$

(A2) $f_{i}(0,0)=0$ and for any $\rho>0$ there exists a constant $k(\rho)>0$ such that

$$
\left|f_{i}\left(u_{1}, v_{1}\right)-f_{i}\left(u_{2}, v_{2}\right)\right| \leq k(\rho)\left[\left(\left|u_{1}\right|^{\alpha}+\left|u_{2}\right|^{\alpha}\right)\left|u_{1}-u_{2}\right|+\left(\left|v_{1}\right|^{\beta}+\left|v_{2}\right|^{\beta}\right)\left|v_{1}-v_{2}\right|\right],
$$

where $\left|u_{i}\right|,\left|v_{i}\right| \leq \rho$, for $u_{i}, v_{i} \in R, i=1,2$, and $0 \leq \alpha \leq \frac{4}{N-4}, 0 \leq \beta \leq \frac{4}{N-4}$.

(A3) $\frac{\partial f_{1}}{\partial v}=\frac{\partial f_{2}}{\partial u}$.

Note the function of the form $f_{1}(u, v)=u^{s-1} v^{s}+u^{p}, f_{2}(u, v)=v^{s-1} u^{s}+v^{q}$ satisfy the assumptions (A1)-(A3) where $1<s, p, q \leq \frac{N}{N-4}$ for $N \geq 4$ or $s, p, q>1$ for $N=1,2,3$. Before proving the existence theorem for nonlinear equations, we will give the definition of weak solution of (1.1)-(1.6) and we need the existence result for a nonhomgeneous wave equation with a strong dissipation.

Definition. A function $w(t)=(u(t), v(t)) \in H_{0}^{1}(\Omega) \times H_{0}^{1}(\Omega), t \in[0, T)$, is called a weak solution of $(1.1)-(1.6)$ if

$$
\left\{\begin{array}{l}
\frac{d}{d t} \int_{\Omega} u_{t} \eta d x=-\int_{\Omega} M\left(\|\nabla u\|_{2}^{2}\right) \nabla u \nabla \eta d x-\int_{\Omega} \nabla u_{t} \nabla \eta d x+\int_{\Omega} f_{1}(u, v) \eta d x \\
\frac{d}{d t} \int_{\Omega} v_{t} \eta d x=-\int_{\Omega} M\left(\|\nabla v\|_{2}^{2}\right) \nabla v \nabla \eta d x-\int_{\Omega} \nabla v_{t} \nabla \eta d x+\int_{\Omega} f_{2}(u, v) \eta d x
\end{array}\right.
$$

holds for any $\eta \in H_{0}^{1}(\Omega)$.

Theorem 3.1. Let $m(t)$ be a nonnegative Lipschitz function and $f(t)$ be a Lipschitz function on $[0, T], T>0$. If $u_{0} \in H^{2}(\Omega) \cap H_{0}^{1}(\Omega)$ and $u_{1} \in L^{2}(\Omega)$, then there exists a unique solution $u$ satisfying

$$
\begin{aligned}
& u(t) \in C^{0}\left([0, T] ; H^{2}(\Omega) \cap H_{0}^{1}(\Omega)\right), \\
& u^{\prime}(t) \in C^{0}\left([0, T] ; L^{2}(\Omega)\right) \cap L^{2}\left((0, T) ; H_{0}^{1}(\Omega)\right),
\end{aligned}
$$

and

$$
\begin{aligned}
u^{\prime \prime}-m(t) \Delta u-\Delta u^{\prime} & =f(t) \text { in } \Omega \times[0, T], \\
u(0) & =u_{0}, u^{\prime}(0)=u_{1}, x \in \Omega, \\
u(x, t) & =0, x \in \partial \Omega, t>0,
\end{aligned}
$$


here $u^{\prime}=\frac{\partial u}{\partial t}$.

Proof. See [17, Prop. 2.2].

Theorem 3.2. Assume (A2) holds and $M(r)$ is a nonnegative locally Lipschitz function for $r \geq 0$ with the Lipschitz constant $L$. If $u_{0}, v_{0} \in H^{2}(\Omega) \cap H_{0}^{1}(\Omega)$ and $u_{1}, v_{1}$ $\in L^{2}(\Omega)$, then there exist a unique local weak solutions $(u, v)$ of (1.1)-(1.6) satisfying

$$
u(t), v(t) \in C^{0}\left([0, T] ; H^{2}(\Omega) \cap H_{0}^{1}(\Omega)\right),
$$

and

$$
u^{\prime}(t), v^{\prime}(t) \in C^{0}\left([0, T] ; L^{2}(\Omega)\right) \cap L^{2}\left((0, T) ; H_{0}^{1}(\Omega)\right), \text { for } T>0 .
$$

Moreover, at least one of the following statements hold:

(i) $T=\infty$

(ii) $e(u(t), v(t)) \equiv\left\|u_{t}\right\|_{2}^{2}+\|\Delta u\|_{2}^{2}+\left\|v_{t}\right\|_{2}^{2}+\|\Delta v\|_{2}^{2} \rightarrow \infty$ as $t \rightarrow T^{-}$.

Proof. We set $w(t)=(u(t), v(t))$, and define the following two-parameter space :

$$
\begin{aligned}
& X_{T, R} \\
& =\left\{\begin{array}{c}
w(t) \in C^{0}\left([0, T] ; H^{2}(\Omega) \cap H_{0}^{1}(\Omega)\right), w_{t}(t) \in C^{0}\left([0, T] ; L^{2}(\Omega)\right) \cap L^{2}\left((0, T) ; H_{0}^{1}(\Omega)\right): \\
e(u(t), v(t)) \leq R^{2}, \text { with } w(0)=\left(u_{0}, v_{0}\right), w_{t}(0)=\left(u_{1}, v_{1}\right) .
\end{array}\right\},
\end{aligned}
$$

for $T>0, R>0$. Then $X_{T, R}$ is a complete metric space with the distance

$$
d(y, z)=\sup _{0 \leq t \leq T}\left\{\left\|(\mu-\varphi)_{t}\right\|_{2}^{2}+\|\Delta \mu-\Delta \varphi\|_{2}^{2}+\left\|(\xi-\psi)_{t}\right\|_{2}^{2}+\|\Delta \xi-\Delta \psi\|_{2}^{2}\right\}^{\frac{1}{2}}
$$

where $y(t)=(\mu(t), \xi(t)), z(t)=(\varphi(t), \psi(t)) \in X_{T, R}$.

Given $\bar{w}(t)=(\bar{u}(t), \bar{v}(t)) \in X_{T, R}$, we consider the linear system

$$
\begin{gathered}
u_{t t}-M\left(\|\nabla \bar{u}\|_{2}^{2}\right) \Delta u-\Delta u_{t}=f_{1}(\bar{u}, \bar{v}) \quad \text { in } \Omega \times[0, T), \\
v_{t t}-M\left(\|\nabla \bar{v}\|_{2}^{2}\right) \Delta v-\Delta v_{t}=f_{2}(\bar{u}, \bar{v}) \quad \text { in } \Omega \times[0, T),
\end{gathered}
$$

with initial conditions,

$$
\begin{aligned}
& u(x, 0)=u_{0}(x), u_{t}(x, 0)=u_{1}(x), \quad x \in \Omega, \\
& v(x, 0)=v_{0}(x), v_{t}(x, 0)=v_{1}(x), \quad x \in \Omega,
\end{aligned}
$$

and boundary conditions,

$$
\begin{aligned}
& u(x, t)=0, x \in \partial \Omega, t>0 \\
& v(x, t)=0, x \in \partial \Omega, t>0 .
\end{aligned}
$$

By Theorem 3.1, there exists a unique solution $w(t)=(u(t), v(t))$ of $(3.3)-(3.8)$. We define the nonlinear mapping $S \bar{w}=w$, and then, we will show that there exist $T>0$ and $R>0$ such that 
(i) $S: X_{T, R} \rightarrow X_{T, R}$,

(ii) $S$ is a contraction mapping in $X_{T, R}$ with respect to the metric $d(\cdot, \cdot)$ defined in (3.2).

Indeed, multiplying (3.3) by $2 u_{t}$, integrating it over $\Omega$, and then by Divergence theorem, we get

$$
\frac{d}{d t}\left\{\left\|u_{t}\right\|_{2}^{2}+M\left(\|\nabla \bar{u}\|_{2}^{2}\right)\|\nabla u\|_{2}^{2}\right\}+2\left\|\nabla u_{t}\right\|_{2}^{2}=I_{u 1}+I_{u 2},
$$

where

$$
\begin{aligned}
I_{u 1} & =\left(\frac{d}{d t} M\left(\|\nabla \bar{u}\|_{2}^{2}\right)\right)\|\nabla u\|_{2}^{2}, \\
I_{u 2} & =\int_{\Omega} 2 f_{1}(\bar{u}, \bar{v}) u_{t} d x .
\end{aligned}
$$

Similarly, we also have

$$
\frac{d}{d t}\left\{\left\|v_{t}\right\|_{2}^{2}+M\left(\|\nabla \bar{v}\|_{2}^{2}\right)\|\nabla v\|_{2}^{2}\right\}+2\left\|\nabla v_{t}\right\|_{2}^{2}=I_{v 1}+I_{v 2},
$$

where

$$
\begin{aligned}
I_{v 1} & =\left(\frac{d}{d t} M\left(\|\nabla \bar{v}\|_{2}^{2}\right)\right)\|\nabla v\|_{2}^{2}, \\
I_{v 2} & =\int_{\Omega} 2 f_{2}(\bar{u}, \bar{v}) v_{t} d x .
\end{aligned}
$$

Note that from Lemma 2.1, Divergence theorem, and $\bar{w} \in X_{T, R}$, we have

$$
\begin{aligned}
\left|I_{u 1}\right| & \leq 2 L\|\Delta \bar{u}\|_{2}\left\|\overline{u_{t}}\right\|_{2}\|\nabla u\|_{2}^{2} \\
& \leq 2 L c_{*}^{2} R^{2}\|\Delta u\|_{2}^{2} \\
& \leq c_{0} L R^{2} e(u, v),
\end{aligned}
$$

where $c_{0}=2 c_{*}^{2}$. In the same way, we get

$$
\left|I_{v 1}\right| \leq c_{0} L R^{2} e(u, v) .
$$

By (3.11), (A2), and Lemma 2.1, we have

$$
\begin{aligned}
\left|I_{u 2}\right| & \leq 2 k \int_{\Omega}\left(|\bar{u}|^{\alpha+1}+|\bar{v}|^{\beta+1}\right)\left|u_{t}\right| d x \\
& \leq 2 k\left[\left(c_{*}\|\Delta \bar{u}\|_{2}\right)^{\alpha+1}+\left(c_{*}\|\Delta \bar{v}\|_{2}\right)^{\beta+1}\right]\left\|u_{t}\right\|_{2} \\
& \leq c_{1}\left(R^{\alpha+1}+R^{\beta+1}\right) e(u, v)^{\frac{1}{2}}
\end{aligned}
$$

where $c_{1}=2 k \max \left(c_{*}^{\alpha+1}, c_{*}^{\beta+1}\right)$. Similarly, we have

$$
\left|I_{v 2}\right| \leq c_{1}\left(R^{\alpha+1}+R^{\beta+1}\right) e(u, v)^{\frac{1}{2}} .
$$


Combining (3.9) and (3.12) together, and by (3.15)-(3.18), we have

$$
\begin{gathered}
\frac{d}{d t}\left\{\left\|u_{t}\right\|_{2}^{2}+M\left(\|\nabla \bar{u}\|_{2}^{2}\right)\|\nabla u\|_{2}^{2}+\left\|v_{t}\right\|_{2}^{2}+M\left(\|\nabla \bar{v}\|_{2}^{2}\right)\|\nabla v\|_{2}^{2}\right\} \\
+2\left\|\nabla u_{t}\right\|_{2}^{2}+2\left\|\nabla v_{t}\right\|_{2}^{2} \\
\leq 2 c_{0} L R^{2} e(u, v)+2 c_{1}\left(R^{\alpha+1}+R^{\beta+1}\right) e(u, v)^{\frac{1}{2}} .
\end{gathered}
$$

On the other hand, multiplying (3.3) by $-2 \Delta u$, and integrating it over $\Omega$, we get

$$
\begin{aligned}
& \frac{d}{d t}\left\{\|\Delta u\|_{2}^{2}-2 \int_{\Omega} u_{t} \Delta u d x\right\}+2 M\left(\|\nabla \bar{u}\|_{2}^{2}\right)\|\Delta u\|_{2}^{2} \\
& \quad=2\left\|\nabla u_{t}\right\|_{2}^{2}-\int_{\Omega} 2 f_{1}(\bar{u}, \bar{v}) \Delta u d x \\
& \quad \leq 2\left\|\nabla u_{t}\right\|_{2}^{2}+c_{1}\left(R^{\alpha+1}+R^{\beta+1}\right) e(u, v)^{\frac{1}{2}}
\end{aligned}
$$

the last inequality in (3.20) is obtained by following the arguments in (3.17). Similarly, we also have

$$
\begin{aligned}
& \frac{d}{d t}\left\{\|\Delta v\|_{2}^{2}-2 \int_{\Omega} v_{t} \Delta v d x\right\}+2 M\left(\|\nabla \bar{v}\|_{2}^{2}\right)\|\Delta v\|_{2}^{2} \\
& \quad \leq 2\left\|\nabla v_{t}\right\|_{2}^{2}+c_{1}\left(R^{\alpha+1}+R^{\beta+1}\right) e(v(t))^{\frac{1}{2}}
\end{aligned}
$$

Now, combining (3.20) and (3.21), we get

$$
\begin{aligned}
\frac{d}{d t}\{ & \left.\|\Delta u\|_{2}^{2}-2 \int_{\Omega} u_{t} \Delta u d x+\|\Delta v\|_{2}^{2}-2 \int_{\Omega} v_{t} \Delta v d x\right\} \\
& +2 M\left(\|\nabla \bar{u}\|_{2}^{2}\right)\|\Delta u\|_{2}^{2}+2 M\left(\|\nabla \bar{v}\|_{2}^{2}\right)\|\Delta v\|_{2}^{2} \\
\leq & 2\left(\left\|\nabla u_{t}\right\|_{2}^{2}+\left\|\nabla v_{t}\right\|_{2}^{2}\right)+2 c_{1}\left(R^{\alpha+1}+R^{\beta+1}\right) e(u, v)^{\frac{1}{2}} .
\end{aligned}
$$

Multiplying (3.22) by $\varepsilon, 0<\varepsilon \leq 1$, and adding (3.19) together, we obtain

$$
\begin{aligned}
& \frac{d}{d t} e_{\bar{u}, \bar{v}}^{*}(u, v)+2(1-\varepsilon)\left[\left\|\nabla u_{t}\right\|_{2}^{2}+\left\|\nabla v_{t}\right\|_{2}^{2}\right] \\
& \quad \leq 2 c_{0} L R^{2} e(u, v)+2(1+\varepsilon) c_{1}\left(R^{\alpha+1}+R^{\beta+1}\right) e(u, v)^{\frac{1}{2}},
\end{aligned}
$$

where

$$
\begin{aligned}
e_{\bar{u}, \bar{v}}^{*}(u, v)= & \left\|u_{t}\right\|_{2}^{2}+\left\|v_{t}\right\|_{2}^{2}+M\left(\|\nabla \bar{u}\|_{2}^{2}\right)\|\nabla u\|_{2}^{2}+M\left(\|\nabla \bar{v}\|_{2}^{2}\right)\|\nabla v\|_{2}^{2} \\
& -2 \varepsilon\left(\int_{\Omega} u_{t} \Delta u d x-\int_{\Omega} v_{t} \Delta v d x\right)+\varepsilon\left(\|\Delta u\|_{2}^{2}+\|\Delta v\|_{2}^{2}\right) .
\end{aligned}
$$

By Young's inequality, we get $\left|2 \varepsilon \int_{\Omega} u_{t} \Delta u d x\right| \leq 2 \varepsilon\left\|u_{t}\right\|_{2}^{2}+\frac{\varepsilon}{2}\|\Delta u\|_{2}^{2}$. Hence

$$
\begin{aligned}
e_{\bar{u}, \bar{v}}^{*}(u, v) \geq & (1-2 \varepsilon)\left(\left\|u_{t}\right\|_{2}^{2}+\left\|v_{t}\right\|_{2}^{2}\right)+\frac{\varepsilon}{2}\left(\|\Delta u\|_{2}^{2}+\|\Delta v\|_{2}^{2}\right) \\
& +M\left(\|\nabla \bar{u}\|_{2}^{2}\right)\|\nabla u\|_{2}^{2}+M\left(\|\nabla \bar{v}\|_{2}^{2}\right)\|\nabla v\|_{2}^{2} .
\end{aligned}
$$


Choosing $\varepsilon=\frac{2}{5}$, we have

$$
e_{\bar{u}, \bar{v}}^{*}(u, v) \geq \frac{1}{5} e(u, v) .
$$

From (3.23) and (3.25), we obtain

$$
\frac{d}{d t} e_{\bar{u}, \bar{v}}^{*}(u(t), v(t)) \leq 10 c_{0} L R^{2} e_{\bar{u}, \bar{v}}^{*}(u(t), v(t))+\frac{14 \sqrt{5}}{5} c_{1}\left(R^{\alpha+1}+R^{\beta+1}\right) e_{\bar{u}, \bar{v}}^{*}(u(t), v(t))^{\frac{1}{2}} .
$$

By Gronwall Lemma, we get

$$
e_{\bar{u}, \bar{v}}^{*}(u(t), v(t)) \leq\left(e_{\bar{u}(0), \bar{v}(0)}^{*}\left(u_{0}, v_{0}\right)^{\frac{1}{2}}+\frac{7 \sqrt{5}}{5} c_{1}\left(R^{\alpha+1}+R^{\beta+1}\right) T\right)^{2} \mathrm{e}^{5 c_{0} L R^{2} T} .
$$

Note that from (3.24), and Young's inequality, we have

$$
e_{\bar{u}(0), \bar{v}(0)}^{*}(u(0), v(0)) \leq c_{2},
$$

where

$$
\begin{aligned}
c_{2}= & 2\left(\left\|u_{1}\right\|_{2}^{2}+\left\|v_{1}\right\|_{2}^{2}\right)+\left\|\Delta u_{0}\right\|_{2}^{2}+\left\|\Delta v_{0}\right\|_{2}^{2} \\
& +M\left(\left\|\nabla u_{0}\right\|_{2}^{2}\right)\left\|\nabla u_{0}\right\|_{2}^{2}+M\left(\left\|\nabla v_{0}\right\|_{2}^{2}\right)\left\|\nabla v_{0}\right\|_{2}^{2} .
\end{aligned}
$$

Then, from $(3.25),(3.26),(3.27)$, we obtain for any $t \in(0, T]$,

$$
\begin{aligned}
e(u(t), v(t)) & \leq 5 e_{\bar{u}, \bar{v}}^{*}(u(t), v(t)) \\
& \leq \chi\left(u_{0}, u_{1}, v_{0}, v_{1}, R, T\right)^{2} \mathrm{e}^{5 c_{0} L R^{2} T},
\end{aligned}
$$

where

$$
\chi\left(u_{0}, u_{1}, v_{0}, v_{1}, R, T\right)=c_{2}^{\frac{1}{2}}+\frac{7 \sqrt{5}}{5} c_{1}\left(R^{\alpha+1}+R^{\beta+1}\right) T .
$$

We see that for parameters $T$ and $R$ satisfy

$$
\chi\left(u_{0}, u_{1}, v_{0}, v_{1}, R, T\right)^{2} \mathrm{e}^{5 c_{0} L R^{2} T} \leq R^{2} .
$$

Then $S$ maps $X_{T, R}$ into itself. By Theorem 3.1, $w \in C^{0}\left([0, T] ; H^{2}(\Omega) \cap H_{0}^{1}(\Omega)\right) \cap$ $C^{1}\left([0, T] ; L^{2}(\Omega)\right)$. Moreover, it follows from (3.19) and (3.29) that $u^{\prime}, v^{\prime} \in L^{2}\left((0, T) ; H_{0}^{1}\right.$ $(\Omega))$. Hence, we see that $u(t)$ and $v(t)$ belong to $C^{0}\left([0, T] ; H^{2}(\Omega) \cap H_{0}^{1}(\Omega)\right) \cap C^{1}\left([0, T] ; L^{2}\right.$ $(\Omega)$ ).

Next, we will show that $S$ is a contraction mapping with respect to the metric $d(\cdot, \cdot)$. Let $\left(\overline{u_{i}}, \overline{v_{i}}\right) \in X_{T, R}$ and $\left(u_{i}, v_{i}\right)$ be the corresponding solution to $(3.3)-(3.8)$. By the above discussion, we see that $\left(u_{i}, v_{i}\right) \in X_{T, R}, i=1$, 2. Setting $w_{1}(t)=\left(u_{1}-u_{2}\right)(t)$, $w_{2}(t)=\left(v_{1}-v_{2}\right)(t)$, then $w_{1}$ and $w_{2}$ satisfy the following system:

$$
\begin{aligned}
& \left(w_{1}\right)_{t t}-M\left(\left\|\nabla \overline{u_{1}}\right\|_{2}^{2}\right) \Delta w_{1}-\Delta\left(w_{1}\right)_{t} \\
& \quad=f_{1}\left(\overline{u_{1}}, \overline{v_{1}}\right)-f_{1}\left(\overline{u_{2}}, \overline{v_{2}}\right)+\left[M\left(\left\|\nabla \overline{u_{1}}\right\|_{2}^{2}\right)-M\left(\left\|\nabla \overline{u_{2}}\right\|_{2}^{2}\right)\right] \Delta u_{2},
\end{aligned}
$$




$$
\begin{gathered}
\left(w_{2}\right)_{t t}-M\left(\left\|\nabla \overline{v_{1}}\right\|_{2}^{2}\right) \Delta w_{2}-\Delta\left(w_{2}\right)_{t} \\
=f_{2}\left(\overline{u_{1}}, \overline{v_{1}}\right)-f_{2}\left(\overline{u_{2}}, \overline{v_{2}}\right)+\left[M\left(\left\|\nabla \overline{v_{1}}\right\|_{2}^{2}\right)-M\left(\left\|\nabla \overline{v_{2}}\right\|_{2}^{2}\right)\right] \Delta v_{2}, \\
w_{1}(0)=0, \quad\left(w_{1}\right)_{t}(0)=0, \\
w_{2}(0)=0, \quad\left(w_{2}\right)_{t}(0)=0 .
\end{gathered}
$$

Multiplying (3.30) by $2\left(w_{1}\right)_{t}$, and integrating it over $\Omega$, we have

$$
\begin{aligned}
& \frac{d}{d t}\left\{\left\|\left(w_{1}\right)_{t}\right\|_{2}^{2}+M\left(\left\|\nabla \overline{u_{1}}\right\|_{2}^{2}\right)\left\|\nabla w_{1}\right\|_{2}^{2}\right\}+2\left\|\nabla\left(w_{1}\right)_{t}\right\|_{2}^{2} \\
& \quad=I_{u 3}+I_{u 4}+I_{u 5},
\end{aligned}
$$

where

$$
\begin{aligned}
I_{u 3} & =\left(\frac{d}{d t} M\left(\left\|\nabla \overline{u_{1}}\right\|_{2}^{2}\right)\right)\left\|\nabla w_{1}\right\|_{2}^{2}, \\
I_{u 4} & =2\left[M\left(\left\|\nabla \overline{u_{1}}\right\|_{2}^{2}\right)-M\left(\left\|\nabla \overline{u_{2}}\right\|_{2}^{2}\right)\right] \int_{\Omega} \Delta u_{2}\left(w_{1}\right)_{t} d x, \\
I_{u 5} & =2 \int_{\Omega}\left(f_{1}\left(\overline{u_{1}}, \overline{v_{1}}\right)-f_{1}\left(\overline{u_{2}}, \overline{v_{2}}\right)\right)\left(w_{1}\right)_{t} d x .
\end{aligned}
$$

Similarly, we have

$$
\begin{aligned}
& \frac{d}{d t}\left\{\left\|\left(w_{2}\right)_{t}\right\|_{2}^{2}+M\left(\left\|\nabla \overline{v_{1}}\right\|_{2}^{2}\right)\left\|\nabla w_{2}\right\|_{2}^{2}\right\}+2\left\|\nabla\left(w_{2}\right)_{t}\right\|_{2}^{2} \\
& \quad=I_{v 3}+I_{v 4}+I_{v 5}
\end{aligned}
$$

where

$$
\begin{aligned}
& I_{v 3}=\left(\frac{d}{d t} M\left(\left\|\nabla \overline{v_{1}}\right\|_{2}^{2}\right)\right)\left\|\nabla w_{2}\right\|_{2}^{2}, \\
& I_{v 4}=2\left[M\left(\left\|\nabla \overline{v_{1}}\right\|_{2}^{2}\right)-M\left(\left\|\nabla \overline{v_{2}}\right\|_{2}^{2}\right)\right] \int_{\Omega} \Delta v_{2}\left(w_{2}\right)_{t} d x \\
& I_{v 5}=2 \int_{\Omega}\left(f_{2}\left(\overline{u_{1}}, \overline{v_{1}}\right)-f_{2}\left(\overline{u_{2}}, \overline{v_{2}}\right)\right)\left(w_{2}\right)_{t} d x .
\end{aligned}
$$

From (3.35), by using the Divergence theorem and Lemma 2.1, we have

$$
\begin{aligned}
\left|I_{u 3}\right| & \leq 2 L\left\|\Delta \overline{u_{1}}\right\|_{2}\left\|\left(\overline{u_{1}}\right)_{t}\right\|_{2}\left\|\nabla w_{1}\right\|_{2}^{2} \\
& \leq c_{0} L R^{2} e\left(w_{1}, w_{2}\right) .
\end{aligned}
$$

Note that by Lemma 2.1, we have

$$
\begin{aligned}
\left|M\left(\left\|\nabla \overline{u_{1}}\right\|_{2}^{2}\right)-M\left(\left\|\nabla \overline{u_{2}}\right\|_{2}^{2}\right)\right| & \leq L\left(\left\|\nabla \overline{u_{1}}\right\|_{2}+\left\|\nabla \overline{u_{2}}\right\|_{2}\right)\left\|\nabla \overline{u_{1}}-\nabla \overline{u_{2}}\right\|_{2} \\
& \leq 2 c_{*}^{2} R L e\left(\overline{u_{1}}-\overline{u_{2}}, \overline{v_{1}}-\overline{v_{2}}\right)^{\frac{1}{2}} .
\end{aligned}
$$


From (3.36) and (3.43), we get

$$
\left|I_{u 4}\right| \leq 4 c_{*}^{2} L R^{2} e\left(\overline{u_{1}}-\overline{u_{2}}, \overline{v_{1}}-\overline{v_{2}}\right)^{\frac{1}{2}} e\left(w_{1}, w_{2}\right)^{\frac{1}{2}} .
$$

By (A2) and Lemma 2.1, we get

$$
\left|I_{u 5}\right| \leq 4 c_{1}\left(R^{\alpha}+R^{\beta}\right) e\left(\overline{u_{1}}-\overline{u_{2}}, \overline{v_{1}}-\overline{v_{2}}\right)^{\frac{1}{2}} e\left(w_{1}, w_{2}\right)^{\frac{1}{2}} .
$$

Hence from (3.34), (3.42), (3.44), (3.45), we have

$$
\begin{aligned}
\frac{d}{d t}\{ & \left.\left\|\left(w_{1}\right)_{t}\right\|_{2}^{2}+M\left(\left\|\nabla \overline{u_{1}}\right\|_{2}^{2}\right)\left\|\nabla w_{1}\right\|_{2}^{2}\right\}+2\left\|\nabla\left(w_{1}\right)_{t}\right\|_{2}^{2} \\
\leq & c_{0} L R^{2} e\left(w_{1}, w_{2}\right)+4 c_{*}^{2} L R^{2} e\left(\overline{u_{1}}-\overline{u_{2}}, \overline{v_{1}}-\overline{v_{2}}\right)^{\frac{1}{2}} e\left(w_{1}, w_{2}\right)^{\frac{1}{2}} \\
& +4 c_{1}\left(R^{\alpha}+R^{\beta}\right) e\left(\overline{u_{1}}-\overline{u_{2}}, \overline{v_{1}}-\overline{v_{2}}\right)^{\frac{1}{2}} e\left(w_{1}, w_{2}\right)^{\frac{1}{2}} .
\end{aligned}
$$

By the same procedure, from (3.38), we have the similar inequality for $w_{2}$. Hence, we obtain

$$
\begin{aligned}
\frac{d}{d t}\{ & \left.\left\|\left(w_{1}\right)_{t}\right\|_{2}^{2}+M\left(\left\|\nabla \overline{u_{1}}\right\|_{2}^{2}\right)\left\|\nabla w_{1}\right\|_{2}^{2}+\left\|\left(w_{2}\right)_{t}\right\|_{2}^{2}+M\left(\left\|\nabla \overline{v_{1}}\right\|_{2}^{2}\right)\left\|\nabla w_{2}\right\|_{2}^{2}\right\} \\
& +2\left\|\nabla\left(w_{1}\right)_{t}\right\|_{2}^{2}+2\left\|\nabla\left(w_{2}\right)_{t}\right\|_{2}^{2} \\
\leq & 2 c_{0} L R^{2} e\left(w_{1}, w_{2}\right)+8 c_{*}^{2} L R^{2} e\left(\overline{u_{1}}-\overline{u_{2}}, \overline{v_{1}}-\overline{v_{2}}\right)^{\frac{1}{2}} e\left(w_{1}, w_{2}\right)^{\frac{1}{2}} \\
& +8 c_{1}\left(R^{\alpha}+R^{\beta}\right) e\left(\overline{u_{1}}-\overline{u_{2}}, \overline{v_{1}}-\overline{v_{2}}\right)^{\frac{1}{2}} e\left(w_{1}, w_{2}\right)^{\frac{1}{2}} .
\end{aligned}
$$

On the other hand, multiplying (3.30) by $-2 \Delta w_{1}$, and integrating it over $\Omega$, and then by Divergence theorem and (A2), we have

$$
\begin{aligned}
\frac{d}{d t}\{ & \left.\left\|\Delta w_{1}\right\|_{2}^{2}-2 \int_{\Omega}\left(w_{1}\right)_{t} \Delta w_{1} d x\right\}+2 M\left(\left\|\nabla \overline{u_{1}}\right\|_{2}^{2}\right)\left\|\Delta w_{1}\right\|_{2}^{2} \\
\leq & 2\left\|\nabla\left(w_{1}\right)_{t}\right\|_{2}^{2}+4 c_{*}^{2} L R^{2} e\left(\overline{u_{1}}-\overline{u_{2}}, \overline{v_{1}}-\overline{v_{2}}\right)^{\frac{1}{2}} e\left(w_{1}, w_{2}\right)^{\frac{1}{2}} \\
& +2 c_{1}\left(R^{\alpha}+R^{\beta}\right) e\left(\overline{u_{1}}-\overline{u_{2}}, \overline{v_{1}}-\overline{v_{2}}\right)^{\frac{1}{2}} e\left(w_{1}, w_{2}\right)^{\frac{1}{2}} .
\end{aligned}
$$

The similar inequality is obtained for $w_{2}$. Therefore, we get

$$
\begin{aligned}
\frac{d}{d t}\{ & \left.\left\|\Delta w_{1}\right\|_{2}^{2}-2 \int_{\Omega}\left(w_{1}\right)_{t} \Delta w_{1} d x+\left\|\Delta w_{2}\right\|_{2}^{2}-2 \int_{\Omega}\left(w_{2}\right)_{t} \Delta w_{2} d x\right\} \\
& +2 M\left(\left\|\nabla \overline{u_{1}}\right\|_{2}^{2}\right)\left\|\Delta w_{1}\right\|_{2}^{2}+2 M\left(\left\|\nabla \overline{v_{1}}\right\|_{2}^{2}\right)\left\|\Delta w_{2}\right\|_{2}^{2} \\
\leq & 2\left[\left\|\nabla\left(w_{1}\right)_{t}\right\|_{2}^{2}+\left\|\nabla\left(w_{2}\right)_{t}\right\|_{2}^{2}\right]+8 c_{*}^{2} L R^{2} e\left(\overline{u_{1}}-\overline{u_{2}}, \overline{v_{1}}-\overline{v_{2}}\right)^{\frac{1}{2}} e\left(w_{1}, w_{2}\right)^{\frac{1}{2}} \\
& +4 c_{1}\left(R^{\alpha}+R^{\beta}\right) e\left(\overline{u_{1}}-\overline{u_{2}}, \overline{v_{1}}-\overline{v_{2}}\right)^{\frac{1}{2}} e\left(w_{1}, w_{2}\right)^{\frac{1}{2}} .
\end{aligned}
$$

Multiplying (3.48) by $\varepsilon, 0<\varepsilon \leq 1$, and adding it to (3.44), we have

$$
\begin{aligned}
\frac{d}{d t} e_{\overline{u_{1}}, \overline{v_{1}}}^{*}\left(w_{1}, w_{2}\right)+2(1-\varepsilon)\left[\left\|\nabla\left(w_{1}\right)_{t}\right\|_{2}^{2}+\left\|\nabla\left(w_{2}\right)_{t}\right\|_{2}^{2}\right] \\
\leq 2 c_{0} L R^{2} e\left(w_{1}, w_{2}\right)+8 c_{*}^{2}(1+\varepsilon) L R^{2} e\left(\overline{u_{1}}-\overline{u_{2}}, \overline{v_{1}}-\overline{v_{2}}\right)^{\frac{1}{2}} e\left(w_{1}, w_{2}\right)^{\frac{1}{2}}+ \\
\quad 4(1+\varepsilon) c_{1}\left(R^{\alpha+1}+R^{\beta+1}\right) e\left(\overline{u_{1}}-\overline{u_{2}}, \overline{v_{1}}-\overline{v_{2}}\right)^{\frac{1}{2}} e\left(w_{1}, w_{2}\right)^{\frac{1}{2}}
\end{aligned}
$$


where $e_{\overline{u_{1}}, \overline{v_{1}}}^{*}\left(w_{1}, w_{2}\right)$ is given by (3.24) with $u=w_{1}, v=w_{2}, \bar{u}=\overline{u_{1}}$ and $\bar{v}=\overline{v_{1}}$. Taking $\varepsilon=\frac{2}{5}$ in (3.49), and as in (3.22)-(3.25), we have

$$
e_{\overline{u_{1}}, \bar{v}_{1}}^{*}\left(w_{1}, w_{2}\right) \geq \frac{1}{5} e\left(w_{1}, w_{2}\right) .
$$

Therefore, from (3.49) and (3.50), we get

$$
\begin{aligned}
\frac{d}{d t} e_{\frac{u_{1}}{u_{1}}, \overline{v_{1}}}^{*}\left(w_{1}, w_{2}\right) \leq & 10 c_{0} L R^{2} e_{\frac{*}{u_{1}}, \overline{v_{1}}}\left(w_{1}, w_{2}\right)+c_{3} L R^{2} e\left(\overline{u_{1}}-\overline{u_{2}}, \overline{v_{1}}-\overline{v_{2}}\right)^{\frac{1}{2}} e_{\overline{u_{1}}, \overline{v_{1}}}^{\frac{*}{1}}\left(w_{1}, w_{2}\right)^{\frac{1}{2}} \\
& +c_{4}\left(R^{\alpha+1}+R^{\beta+1}\right) e\left(\overline{u_{1}}-\overline{u_{2}}, \overline{v_{1}}-\overline{v_{2}}\right)^{\frac{1}{2}} e_{\overline{u_{1}}}^{*}, \overline{v_{1}}\left(w_{1}, w_{2}\right)^{\frac{1}{2}},
\end{aligned}
$$

where $c_{3}=\frac{56 \sqrt{5}}{5} c_{*}^{2}, c_{4}=\frac{28 \sqrt{5}}{5} c_{1}$. Noting that $e_{\overline{u_{1}}(0), \overline{v_{1}}(0)}^{*}\left(w_{1}(0), w_{2}(0)\right)=0$, and by applying Gronwall Lemma, we get

$e_{\overline{u_{1}}, \overline{v_{1}}}^{*}\left(w_{1}, w_{2}\right) \leq\left[\frac{c_{3}}{2} L R^{2}+\frac{c_{4}}{2}\left(R^{\alpha+1}+R^{\beta+1}\right)\right]^{2} T^{2} \mathrm{e}^{5 c_{0} L R^{2} T} \sup _{0 \leq t \leq T} e\left(\overline{u_{1}}-\overline{u_{2}}, \overline{v_{1}}-\overline{v_{2}}\right)$.

By (3.2) and (3.50), we have

$$
d\left(\left(u_{1}, v_{1}\right),\left(u_{2}, v_{2}\right)\right) \leq C(T, R)^{\frac{1}{2}} d\left(\left(\overline{u_{1}}, \overline{v_{1}}\right),\left(\overline{u_{2}}, \overline{v_{2}}\right)\right),
$$

where

$$
C(T, R)=5\left[\frac{c_{3}}{2} L R^{2}+\frac{c_{3}}{2}\left(R^{\alpha+1}+R^{\beta+1}\right)\right] T \mathrm{e}^{5 c_{0} L R^{2} T} .
$$

Hence, under inequality (3.29), $S$ is a contraction mappingn if $C(T, R)<1$. Indeed, we choose $R$ sufficient large and $T$ sufficient small so that (3.29) and (3.51) are satisfied at the same time. By applying Banach fixed point theorem, we obtain the local existence result.

\section{Blow-up property}

In this section, we will study blow-up phenomena of solutions for a system (1.1)-(1.6). In order to state our results, we further make the following assumptions:

(A4) there exists a positive constant $\delta>0$ such that

$$
u f_{1}(u, v)+v f_{2}(u, v) \geq(2+4 \delta) F(u, v), \text { for all } u, v \in R,
$$

where $F(u, v)$ is given in (A1).

(A5) $(2 \delta+1) \bar{M}(s) \geq M(s) s$, for all $s \geq 0$, and $\delta$ is the constant given in (A4), where $\bar{M}(s)=\int_{0}^{s} M(r) d r$.

Definition. A solution $w(t)=(u(t), v(t))$ of $(1.1)-(1.6)$ is called blow-up if there exists a finite time $T^{*}$ such that

$$
\lim _{t \rightarrow T^{*-}} \int_{\Omega}\left(|\nabla u|^{2}+|\nabla v|^{2}\right) d x=\infty .
$$


Let $(u(t), v(t))$ be the solution of (1.1)-(1.6), define the energy function

$$
E(t)=\frac{1}{2}\left[\left\|u_{t}\right\|_{2}^{2}+\left\|v_{t}\right\|_{2}^{2}+\bar{M}\left(\|\nabla u\|_{2}^{2}\right)+\bar{M}\left(\|\nabla v\|_{2}^{2}\right)\right]-\int_{\Omega} F(u, v) d x, t \geq 0 .
$$

Lemma 4.1. Assume that (A1) and (A3) hold, then $E(t)$ is a nonincreasing function and

$$
E(t)=E(0)-\int_{0}^{t}\left(\left\|\nabla u_{t}\right\|_{2}^{2}+\left\|\nabla v_{t}\right\|_{2}^{2}\right) d t
$$

Proof. By differentiating (4.1) and using (1.1), (1.2), (A1) and (A3), we get

$$
\frac{d E(t)}{d t}=-\left(\left\|\nabla u_{t}\right\|_{2}^{2}+\left\|\nabla v_{t}\right\|_{2}^{2}\right)
$$

Thus, Lemma 4.1 follows at once.

Now, let

$$
a(t)=\int_{\Omega}\left(u^{2}+v^{2}\right) d x+\int_{0}^{t}\left(\|\nabla u\|_{2}^{2}+\|\nabla v\|_{2}^{2}\right) d t, t \geq 0 .
$$

Lemma 4.2. Assume that (A1), (A3), (A4) and (A5) hold, we have

$$
\begin{aligned}
& a^{\prime \prime}(t)-4(\delta+1) \int_{\Omega}\left(u_{t}^{2}+v_{t}^{2}\right) d x \\
& \quad \geq(-4-8 \delta) E(0)+(4+8 \delta) \int_{0}^{t}\left(\left\|\nabla u_{t}\right\|_{2}^{2}+\left\|\nabla v_{t}\right\|_{2}^{2}\right) d t .
\end{aligned}
$$

Proof. Form (4.3), we have

$$
a^{\prime}(t)=2 \int_{\Omega}\left(u u_{t}+v v_{t}\right) d x+\|\nabla u\|_{2}^{2}+\|\nabla v\|_{2}^{2} .
$$

By (1.1), (1.2) and Divergence theorem, we get

$$
\begin{aligned}
a^{\prime \prime}(t)= & 2 \int_{\Omega}\left(u_{t}^{2}+v_{t}^{2}\right) d x-2\left(M\left(\|\nabla u\|_{2}^{2}\right)\|\nabla u\|_{2}^{2}+M\left(\|\nabla v\|_{2}^{2}\right)\|\nabla v\|_{2}^{2}\right) \\
& +2 \int_{\Omega}\left(u f_{1}(u, v)+v f_{2}(u, v)\right) d x .
\end{aligned}
$$


By (4.2), we have from (4.6)

$$
\begin{aligned}
a^{\prime \prime}(t) & -4(\delta+1) \int_{\Omega}\left(u_{t}^{2}+v_{t}^{2}\right) d x \\
= & (-4-8 \delta) E(0)+(4+8 \delta) \int_{0}^{t}\left(\left\|\nabla u_{t}\right\|_{2}^{2}+\left\|\nabla v_{t}\right\|_{2}^{2}\right) d s \\
+ & {\left[(2+4 \delta) \bar{M}\left(\|\nabla u\|_{2}^{2}\right)-2 M\left(\|\nabla u\|_{2}^{2}\right) \int_{\Omega}|\nabla u|^{2} d x\right] } \\
+ & {\left[(2+4 \delta) \bar{M}\left(\|\nabla v\|_{2}^{2}\right)-2 M\left(\|\nabla v\|_{2}^{2}\right) \int_{\Omega}|\nabla v|^{2} d x\right] } \\
& +\int_{\Omega} 2\left[u f_{1}(u, v)+v f_{2}(u, v)-(2+4 \delta) F(u, v)\right] d x .
\end{aligned}
$$

Therefore, from (A4) and (A5), we obtain (4.4). Now, we consider three different cases on the sign of the initial energy $E(0)$.(1) If $E(0)<0$, then from (4.4), we have

$$
a^{\prime}(t) \geq a^{\prime}(0)-4(1+2 \delta) E(0) t, t \geq 0
$$

Thus we get $a^{\prime}(t)>\left\|\nabla u_{0}\right\|_{2}^{2}+\left\|\nabla v_{0}\right\|_{2}^{2}$ for $t>t^{*}$, where

$$
t^{*}=\max \left\{\frac{a^{\prime}(0)-\left(\left\|\nabla u_{0}\right\|_{2}^{2}+\left\|\nabla v_{0}\right\|_{2}^{2}\right)}{4(1+2 \delta) E(0)}, 0\right\} .
$$

(2) If $E(0)=0$, then $a^{\prime \prime}(t) \geq 0$ for $t \geq 0$. If $a^{\prime}(0)>\left\|\nabla u_{0}\right\|_{2}^{2}+\left\|\nabla v_{0}\right\|_{2}^{2}$, then we have $a^{\prime}(t)>\left\|\nabla u_{0}\right\|_{2}^{2}+\left\|\nabla v_{0}\right\|_{2}^{2}, t \geq 0$.(3) For the case that $E(0)>0$, we first note that

$$
2 \int_{0}^{t} \int_{\Omega} \nabla u \nabla u_{t} d x d t=\|\nabla u\|_{2}^{2}-\left\|\nabla u_{0}\right\|_{2}^{2}
$$

By Hölder inequality and Young's inequality, we have from (4.8)

$$
\|\nabla u\|_{2}^{2} \leq\left\|\nabla u_{0}\right\|_{2}^{2}+\int_{0}^{t}\|\nabla u\|_{2}^{2} d t+\int_{0}^{t}\left\|\nabla u_{t}\right\|_{2}^{2} d t
$$

In the same way, we get

$$
\|\nabla v\|_{2}^{2} \leq\left\|\nabla v_{0}\right\|_{2}^{2}+\int_{0}^{t}\|\nabla v\|_{2}^{2} d t+\int_{0}^{t}\left\|\nabla v_{t}\right\|_{2}^{2} d t
$$

By Hölder inequality, Young's inequality and then using (4.9) and (4.10), we have from (4.5)

$$
a^{\prime}(t) \leq a(t)+\left\|\nabla u_{0}\right\|_{2}^{2}+\left\|\nabla v_{0}\right\|_{2}^{2}+\int_{\Omega}\left(u_{t}^{2}+v_{t}^{2}\right) d x+\int_{0}^{t}\left(\left\|\nabla u_{t}\right\|_{2}^{2}+\left\|\nabla v_{t}\right\|_{2}^{2}\right) d t .
$$


Hence by (4.4) and (4.11), we obtain

$$
a^{\prime \prime}(t)-4(\delta+1) a^{\prime}(t)+4(\delta+1) a(t)+K_{1} \geq 0,
$$

where

$$
K_{1}=(4+8 \delta) E(0)+4(\delta+1)\left(\left\|\nabla u_{0}\right\|_{2}^{2}+\left\|\nabla v_{0}\right\|_{2}^{2}\right)
$$

Let

$$
b(t)=a(t)+\frac{K_{1}}{4(1+\delta)}, t>0 .
$$

Then $b(t)$ satisfies (2.2). By Lemma 2.2, we see that if

$$
a^{\prime}(0)>r_{2}\left[a(0)+\frac{K_{1}}{4(1+\delta)}\right]+\left(\left\|\nabla u_{0}\right\|_{2}^{2}+\left\|\nabla v_{0}\right\|_{2}^{2}\right),
$$

then $a^{\prime}(t)>\left(\left\|\nabla u_{0}\right\|_{2}^{2}+\left\|\nabla v_{0}\right\|_{2}^{2}\right), t>0$, where $r_{2}$ is given in Lemma 2.2. Consequently, we have

Lemma 4.3. Assume that (A1), (A3), (A4) and (A5) hold and that either one of the following statements is satisfied:

(i) $E(0)<0$,

(ii) $E(0)=0$ and $a^{\prime}(0)>\left(\left\|\nabla u_{0}\right\|_{2}^{2}+\left\|\nabla v_{0}\right\|_{2}^{2}\right)$,

(iii) $E(0)>0$ and (4.12) holds,

then $a^{\prime}(t)>\left(\left\|\nabla u_{0}\right\|_{2}^{2}+\left\|\nabla v_{0}\right\|_{2}^{2}\right)$ for $t>t_{0}$, where $t_{0}=t^{*}$ is given by $(4.7)$ in case (i) and $t_{0}=0$ in cases (ii) and (iii).

Now, we will find the estimate for the life span of $a(t)$. Let

$$
J(t)=\left[a(t)+\left(T_{1}-t\right)\left(\left\|\nabla u_{0}\right\|_{2}^{2}+\left\|\nabla v_{0}\right\|_{2}^{2}\right)\right]^{-\delta}, \text { for } t \in\left[0, T_{1}\right]
$$

where $T_{1}>0$ is a certain constant which will be specified later. Then we have

$$
J^{\prime}(t)=-\delta J(t)^{1+\frac{1}{\delta}}\left(a^{\prime}(t)-\left\|\nabla u_{0}\right\|_{2}^{2}-\left\|\nabla v_{0}\right\|_{2}^{2}\right)
$$

and

$$
J^{\prime \prime}(t)=-\delta J(t)^{1+\frac{2}{\delta}} V(t)
$$

where

$$
\begin{aligned}
V(t)= & a^{\prime \prime}(t)\left[a(t)+\left(T_{1}-t\right)\left(\left\|\nabla u_{0}\right\|_{2}^{2}+\left\|\nabla v_{0}\right\|_{2}^{2}\right)\right] \\
& -(1+\delta)\left(a^{\prime}(t)-\left\|\nabla u_{0}\right\|_{2}^{2}-\left\|\nabla v_{0}\right\|_{2}^{2}\right)^{2} .
\end{aligned}
$$


For simplicity of calculation, we denote

$$
\begin{aligned}
P_{u} & =\int_{\Omega} u^{2} d x, P_{v}=\int_{\Omega} v^{2} d x, \\
Q_{u} & =\int_{0}^{t}\|\nabla u\|_{2}^{2} d t, Q_{v}=\int_{0}^{t}\|\nabla v\|_{2}^{2} d t, \\
R_{u} & =\int_{\Omega} u_{t}^{2} d x, R_{v}=\int_{\Omega} v_{t}^{2} d x, \\
S_{u} & =\int_{0}^{t}\left\|\nabla u_{t}\right\|_{2}^{2} d t, S_{v}=\int_{0}^{t}\left\|\nabla v_{t}\right\|_{2}^{2} d t .
\end{aligned}
$$

From (4.5), (4.8), and Hölder inequality, we get

$$
\begin{aligned}
a^{\prime}(t)= & 2 \int_{\Omega}\left(u u_{t}+v v_{t}\right) d x+\left\|\nabla u_{0}\right\|_{2}^{2}+\left\|\nabla v_{0}\right\|_{2}^{2} \\
& +2 \int_{0}^{t} \int_{\Omega} \nabla u \nabla u_{t} d x d t+2 \int_{0}^{t} \int_{\Omega} \nabla v \nabla v_{t} d x d t \\
\leq & 2\left(\sqrt{R_{u} P_{u}}+\sqrt{Q_{u} S_{u}}+\sqrt{R_{v} P_{v}}+\sqrt{Q_{v} S_{v}}\right) \\
& +\left\|\nabla u_{0}\right\|_{2}^{2}+\left\|\nabla v_{0}\right\|_{2}^{2} .
\end{aligned}
$$

By (4.4), we have

$$
a^{\prime \prime}(t) \geq(-4-8 \delta) E(0)+4(1+\delta)\left(R_{u}+S_{u}+R_{v}+S_{v}\right) .
$$

Thus, from (4.16), (4.17), (4.15) and (4.13), we obtain

$$
\begin{aligned}
V(t) \geq & {\left[(-4-8 \delta) E(0)+4(1+\delta)\left(R_{u}+S_{u}+R_{v}+S_{v}\right)\right] J(t)^{-\frac{1}{\delta}} } \\
& -4(1+\delta)\left(\sqrt{R_{u} P_{u}}+\sqrt{Q_{u} S_{u}}+\sqrt{R_{v} P_{v}}+\sqrt{Q_{v} S_{v}}\right)^{2} .
\end{aligned}
$$

And by (4.13) and (4.3), we have

$$
\begin{aligned}
V(t) \geq & (-4-8 \delta) E(0) J(t)^{-\frac{1}{\delta}} \\
& +4(1+\delta)\left[\left(R_{u}+S_{u}+R_{v}+S_{v}\right)\left(T_{1}-t\right)\left(\left\|\nabla u_{0}\right\|_{2}^{2}+\left\|\nabla v_{0}\right\|_{2}^{2}\right)+\Theta(t)\right],
\end{aligned}
$$

where

$$
\begin{aligned}
\Theta(t)= & \left(R_{u}+S_{u}+R_{v}+S_{v}\right)\left(P_{u}+Q_{u}+P_{v}+Q_{v}\right) \\
& -\left(\sqrt{R_{u} P_{u}}+\sqrt{Q_{u} S_{u}}+\sqrt{R_{v} P_{v}}+\sqrt{Q_{v} S_{v}}\right)^{2} .
\end{aligned}
$$

By Schwarz inequality, $\Theta(t)$ is nonnegative. Hence, we have

$$
V(t) \geq(-4-8 \delta) E(0) J(t)^{-\frac{1}{\delta}}, t \geq t_{0} .
$$


Therefore by (4.14) and (4.18), we get

$$
J^{\prime \prime}(t) \leq \delta(4+8 \delta) E(0) J(t)^{1+\frac{1}{\delta}}, t \geq t_{0} .
$$

Note that by Lemma $4.3, J^{\prime}(t)<0$ for $t>t_{0}$. Mutiplying (4.19) by $J^{\prime}(t)$ and integrating it from $t_{0}$ to $t$, we get

$$
J^{\prime}(t)^{2} \geq \alpha+\beta J(t)^{2+\frac{1}{\delta}} \text { for } t \geq t_{0},
$$

where

$$
\alpha=\delta^{2} J\left(t_{0}\right)^{2+\frac{2}{\delta}}\left[\left(a^{\prime}\left(t_{0}\right)-\left\|\nabla u_{0}\right\|_{2}^{2}-\left\|\nabla v_{0}\right\|_{2}^{2}\right)^{2}-8 E(0) J\left(t_{0}\right)^{\frac{-1}{\delta}}\right],
$$

and

$$
\beta=8 \delta^{2} E(0)
$$

We observe that

$$
\alpha>0 \text { iff } E(0)<\frac{\left(a^{\prime}\left(t_{0}\right)-\left\|\nabla u_{0}\right\|_{2}^{2}-\left\|\nabla v_{0}\right\|_{2}^{2}\right)^{2}}{8\left[a\left(t_{0}\right)+\left(T_{1}-t_{0}\right)\left(\left\|\nabla u_{0}\right\|_{2}^{2}+\left\|\nabla v_{0}\right\|_{2}^{2}\right)\right]} .
$$

Then by Lemma 2.3 , there exists a finite time $T^{*}$ such that $\lim _{t \rightarrow T^{*}-} J(t)=0$ and the upper bound of $T^{*}$ is estimated respectively according to the sign of $E(0)$. This means that

$$
\lim _{t \rightarrow T^{*-}}\left\{\int_{\Omega}\left(u^{2}+v^{2}\right) d x+\int_{0}^{t}\left(\|\nabla u\|_{2}^{2}+\|\nabla v\|_{2}^{2}\right) d t\right\}=\infty .
$$

By Poincaré inequality, it implies that

$$
\lim _{t \rightarrow T^{*-}} \int_{\Omega}\left(|\nabla u|^{2}+|\nabla v|^{2}\right) d x=\infty
$$

Theorem 4.4. Assume that (A1), (A3), (A4) and (A5) hold and that either one of the following statements is satisfied:

(i) $E(0)<0$,

(ii) $E(0)=0$ and $a^{\prime}(0)>\left(\left\|\nabla u_{0}\right\|_{2}^{2}+\left\|\nabla v_{0}\right\|_{2}^{2}\right)$,

(iii) $0<E(0)<\frac{\left(a^{\prime}\left(t_{0}\right)-\left\|\nabla u_{0}\right\|_{2}^{2}-\left\|\nabla v_{0}\right\|_{2}^{2}\right)^{2}}{8\left[a\left(t_{0}\right)+\left(T_{1}-t_{0}\right)\left(\left\|\nabla u_{0}\right\|_{2}^{2}+\left\|\nabla v_{0}\right\|_{2}^{2}\right)\right]}$ and (4.12) holds,

then the solution $(u(t), v(t))$ blows up at finite time $T^{*}$ in the sense of (4.22).

In case (i),

$$
T^{*} \leq t_{0}-\frac{J\left(t_{0}\right)}{J^{\prime}\left(t_{0}\right)} .
$$

Furthermore, if $J\left(t_{0}\right)<\min \left\{1, \sqrt{\frac{\alpha}{-\beta}}\right\}$, we have

$$
T^{*} \leq t_{0}+\frac{1}{\sqrt{-\beta}} \ln \frac{\sqrt{\frac{\alpha}{-\beta}}}{\sqrt{\frac{\alpha}{-\beta}}-J\left(t_{0}\right)} .
$$


In case (ii),

$$
T^{*} \leq t_{0}-\frac{J\left(t_{0}\right)}{J^{\prime}\left(t_{0}\right)}
$$

or

$$
T^{*} \leq t_{0}+\frac{J\left(t_{0}\right)}{\sqrt{\alpha}}
$$

In case (iii),

$$
T^{*} \leq \frac{J\left(t_{0}\right)}{\sqrt{\alpha}}
$$

or

$$
T^{*} \leq t_{0}+2^{\frac{3 \delta+1}{2 \delta}} \frac{\delta c}{\sqrt{\alpha}}\left\{1-\left[1+c J\left(t_{0}\right)\right]^{\frac{-1}{2 \delta}}\right\}
$$

where $c=\left(\frac{\alpha}{\beta}\right)^{2+\frac{1}{\delta}}$, here $\alpha$ and $\beta$ are given in (4.20), (4.21). Note that in case $(i), t_{0}=t^{*}$ is given in (4.7) and $t_{0}=0$ in case (ii) and (iii). The choice of $T_{1}$ in (4.13) is possible under some conditions. We shall discuss it in the following Remark.

\section{Remark 4.5.}

(i) For the case $E(0)=0$

(1) If $2 \delta \int_{\Omega}\left(u_{0} u_{1}+v_{0} v_{1}\right) d x-\left(\left\|\nabla u_{0}\right\|_{2}^{2}+\left\|\nabla v_{0}\right\|_{2}^{2}\right)>0$, by (4.25), we choose

$$
T_{1} \geq-\frac{J(0)}{J^{\prime}(0)}
$$

Then, in particular, we have

$$
T^{*} \leq T_{1}=\omega
$$

where

$$
\omega=\frac{\left\|u_{0}\right\|_{2}^{2}+\left\|v_{0}\right\|_{2}^{2}}{2 \delta \int_{\Omega}\left(u_{0} u_{1}+v_{0} v_{1}\right) d x-\left(\left\|\nabla u_{0}\right\|_{2}^{2}+\left\|\nabla v_{0}\right\|_{2}^{2}\right)} .
$$

(2) If $0<2 \delta \int_{\Omega}\left(u_{0} u_{1}+v_{0} v_{1}\right) d x \leq\left(\left\|\nabla u_{0}\right\|_{2}^{2}+\left\|\nabla v_{0}\right\|_{2}^{2}\right)$, by (4.26), we choose

$$
T_{1} \geq \frac{J(0)}{\sqrt{\alpha}} .
$$

From (4.29), and by Young's inequality, we get

$$
\begin{aligned}
& \left\|u_{0}\right\|_{2}^{2}+\left\|v_{0}\right\|_{2}^{2}+T_{1}\left(\left\|\nabla u_{0}\right\|_{2}^{2}+\left\|\nabla v_{0}\right\|_{2}^{2}\right) \\
& \quad \leq \delta T_{1}\left(\left\|u_{0}\right\|_{2}^{2}+\left\|u_{1}\right\|_{2}^{2}+\left\|v_{0}\right\|_{2}^{2}+\left\|v_{1}\right\|_{2}^{2}\right), \\
& \quad \leq \bar{c} T_{1}\left(\left\|\nabla u_{0}\right\|_{2}^{2}+\left\|\nabla v_{0}\right\|_{2}^{2}+\left\|u_{1}\right\|_{2}^{2}+\left\|v_{1}\right\|_{2}^{2}\right),
\end{aligned}
$$


where the last inequality is obtained by Lemma 2.1 , with $\bar{c}=\max \left(\delta c_{*}^{2}, 1\right)$. Then, in particular, we have

$$
T^{*} \leq T_{1}=\rho,
$$

where

$$
\rho=\frac{\left\|u_{0}\right\|_{2}^{2}+\left\|v_{0}\right\|_{2}^{2}}{(\bar{c}-1)\left[\left\|\nabla u_{0}\right\|_{2}^{2}+\left\|\nabla v_{0}\right\|_{2}^{2}\right]+\bar{c}\left(\left\|u_{1}\right\|_{2}^{2}+\left\|v_{1}\right\|_{2}^{2}\right)} .
$$

(ii) For the case $E(0)<0$

(1) If $\int_{\Omega}\left(u_{0} u_{1}+v_{0} v_{1}\right) d x>0$, then $a^{\prime}(t)>\left\|\nabla u_{0}\right\|_{2}^{2}+\left\|\nabla v_{0}\right\|_{2}^{2}$ and $t^{*}=0$ (by (4.7)). Thus $T_{1}$ can be chosen as in (i).

(2) If $\int_{\Omega}\left(u_{0} u_{1}+v_{0} v_{1}\right) d x \leq 0$, then $t^{*}=\frac{a^{\prime}(0)-\left\|\nabla u_{0}\right\|_{2}^{2}-\left\|\nabla v_{0}\right\|_{2}^{2}}{4(1+2 \delta) E(0)}$. Thus, by (4.23), we choose $T_{1} \geq t^{*}-\frac{J\left(t^{*}\right)}{J^{\prime}\left(t^{*}\right)}$.

(iii) For the case $E(0)>0$ By (4.27), we choose

$$
T_{1}=\frac{\left\|u_{0}\right\|_{2}^{2}+\left\|v_{0}\right\|_{2}^{2}}{\left[c \delta r_{2}^{2}\left(\left\|\nabla u_{0}\right\|_{2}^{2}+\left\|\nabla v_{0}\right\|_{2}^{2}\right)-1\right]\left(\left\|\nabla u_{0}\right\|_{2}^{2}+\left\|\nabla v_{0}\right\|_{2}^{2}\right)},
$$

here

$$
\begin{aligned}
& c=1, \quad \text { if }\left\|\nabla u_{0}\right\|_{2}^{2}+\left\|\nabla v_{0}\right\|_{2}^{2}>\frac{1}{\delta r_{2}^{2}}, \\
& c=\frac{2}{\delta r_{2}^{2}\left(\left\|\nabla u_{0}\right\|_{2}^{2}+\left\|\nabla v_{0}\right\|_{2}^{2}\right)}, \quad \text { if }\left\|\nabla u_{0}\right\|_{2}^{2}+\left\|\nabla v_{0}\right\|_{2}^{2} \leq \frac{1}{\delta r_{2}^{2}} .
\end{aligned}
$$

Under the condition

$$
E(0)<\min \left\{\kappa_{1}, \kappa_{2}\right\}
$$

where

$$
\kappa_{1}=\frac{(1+\delta)\left[a^{\prime}(0)-r_{2} a(0)-\left(r_{2}+1\right)\left(\left\|\nabla u_{0}\right\|_{2}^{2}+\left\|\nabla v_{0}\right\|_{2}^{2}\right)\right]}{r_{2}(1+2 \delta)}
$$

and

$$
\kappa_{2}=\frac{\left(\int_{\Omega}\left(u_{0} u_{1}+v_{0} v_{1}\right) d x\right)^{2}\left[c \delta r_{2}^{2}\left(\left\|\nabla u_{0}\right\|_{2}^{2}+\left\|\nabla v_{0}\right\|_{2}^{2}\right)-1\right]}{2 c \delta r_{2}^{2}\left(\left\|u_{0}\right\|_{2}^{2}+\left\|v_{0}\right\|_{2}^{2}\right)\left(\left\|\nabla u_{0}\right\|_{2}^{2}+\left\|\nabla v_{0}\right\|_{2}^{2}\right)} .
$$

Then, we have

$$
T^{*} \leq \frac{\mu}{\sqrt{4\left(\int_{\Omega} u_{0} u_{1}+v_{0} v_{1} d x\right)^{2}-8 E(0) \mu}},
$$

where

$$
\mu=\frac{c \delta r_{2}^{2}\left(\left\|u_{0}\right\|_{2}^{2}+\left\|v_{0}\right\|_{2}^{2}\right)\left(\left\|\nabla u_{0}\right\|_{2}^{2}+\left\|\nabla v_{0}\right\|_{2}^{2}\right)}{c \delta r_{2}^{2}\left(\left\|\nabla u_{0}\right\|_{2}^{2}+\left\|\nabla v_{0}\right\|_{2}^{2}\right)-1} .
$$




\section{References}

[1] J. J. Bae, On coupled wave equation of Kirchhoff type with nonlinear boundary damping and memory term, Applied Math. and Computation, 129(2002), 87-105.

[2] A. Benaissa and S. A. Messaoudi, Blow-up of solutions of a quasilinear wave equation with nonlinear dissipation, J. Partial Diff. Eqns. 15(2002), 61-67.

[3] A. Benaissa and S. A. Messaoudi, Blow-up of solutions for the Kirchhoff equation of qLaplacian type with nonlinear dissipation, Colloquium Mathematicum, 94(2002), 103-109.

[4] E. H. Brito, Nonlinear initial boundary value problems, Nonlinear Anal. Theory, Methods, Appliciations 11(1987), 125-137.

[5] D. Del Santo, V. Georgiev and E. Mitidieri, Global existence of the solutions and formation of singularities for a class of hyperbolic systems, Geometric optics and related topics, F. colombini and N. Llerner, Ed. Progress in nonlinear differential equations and their applications, (1997), 117-140.

[6] K. Deng, Blow-up of solutions of some nonlinear hyperbolic systems, Rocky Moutain J. of Math. 29(1999), 807-820.

[7] K. Deng, Nonexistence of global solutions of a nonlinear hyperbolic system, Transactions of The American Math. Society, 349(1997), 1685-1696.

[8] M. Hosoya and Y. Yamada, On some nonlinear wave equations II : global existence and energy decay of solutions, J. Fac. Sci. Univ. Toyko Sect. IA Math. 38(1991), 239-250.

[9] R. Ikehata, On the existence of global solutions for some nonlinear hyperbolic equations with Neumann conditions, TRU Mathematics 24(1988), 1-7.

[10] Ikehata, R., A note on the global solvability of solutions to some nonlinear wave equations with dissipative terms, Differential and Integral Equations 8(1995), 607-616.

[11] G. Kirchhoff, Vorlesungen über Mechanik, Leipzig, Teubner, 1883.

[12] M. R. Li and L. Y. Tsai, Existence and nonexistence of global solutions of some systems of semilinear wave equations, Nonlinear Anal., Theory, Methods and Applications 54(2003), $1397-1415$

[13] T. Matsuyama and R. Ikehata, On global solutions and energy decay for the wave equations of the Kirchhoff type with nonlinear damping terms, J. of Math. Anal and Appli, 204(1996), $729-753$.

[14] K. Nishihara and Y. Yamada, On global solutions of some degenerate quasilinear hyperbolic equations with dissipative terms, Funkcial Ekvac. 33(1990), 151-159.

[15] M. Ohta, Remarks on blow up of solutions for nonlinear evolution equations of second order, Advances in Math. Sci. and Appli. Gakkotosho, Toyko, 2(1998), 901-910.

[16] K. Ono, Global existence, decay, and blowup of solutions for some mildly degenerate nonlinear Kirchhoff strings, J. Diff. Eqns., 137(1997), 273-301.

[17] K. Ono, On global existence, asymptotic stability and blowing up of solutions for some degenerate nonlinear wave equations of Kirchhoff type with a strong dissipation, Math. Methods in The Appl. Sci. 20(1997), 151-177.

[18] K. Ono, On global solutions and blow-up solutions of nonlinear Kirchhoff strings with nonlinear dissipation, J. of Math. Anal. and Appli., 216(1997), 321-342.

[19] J. Y. Park and J. J. Bae, On existence of solutions of nondegenertaer wave equations with nonlinear damping terms, Nihonkai Math. J. 9(1998), 27-46.

[20] J. Y. Park and J. J. Bae, Variational inequality for quasilinear wave equations with nonlinear damping terms, Nonlinear Anal. Theory, Methods, Application, to appear. 
[21] J. Y. Park and J. J. Bae, On existence of solutions of degenertaer wave equations with nonlinear damping terms, J. Korean Math. Soc., 35(1998), 465-490.

[22] S. T. Wu and L. Y. Tsai, Blow-up of solutions for some nonlinear wave equations of Kirchhoff type with some dissipation, Nonlinear Analysis 65(2006), 243-264.

General Educational Center, China University of Technology, Taipei, TAIWAN 116.

E-mail: stwu@cute.edu.tw

Department of Mathematical Science, National Chengchi University, Taipei, TAIWAN 116. 